\title{
Caracterización de las principales tendencias de la gastronomía mexicana en el marco de nuevos escenarios sociales
}

Recibido: 14/10/2016 · Aceptado: 28/10/2016

\author{
Andrés López Ojeda* \\ Universidad Autónoma del Estado de México \\ Carmen Pérez Camacho \\ C2 Cultura y Ciudadanía A. C. \\ Celia Guzmán Hernández \\ Ricardo Hernández López \\ Universidad Autónoma del Estado de México
}

\section{Resumen}

La gastronomía en México se ha consolidado como un importante campo para el desarrollo y se ha colocado a la vanguardia a nivel internacional; contribuye al fortalecimiento de las relaciones sociales y la gastronomía tradicional y popular es un factor de identidad y distintivo cultural. En este marco, uno de los objetivos del trabajo consiste en analizar las principales tendencias gastronómicas, las cuales se caracterizan por su heterogeneidad, pero también por formar parte de procesos más amplios, entre los que destaca la globalización, el esteticismo de la oferta y la aparición de nuevos estilos de vida y consumo. Por lo tanto, se hace necesario fortalecer el conocimiento del sector gastronómico para entender su desarrollo y proyección, particularmente el campo de la profesionalización y la investigación que se vislumbra como imprescindible, sobre todo, en lo referente al mayor conocimiento de las propias gastronomías (revaloración, visibilidad, conservación de tradiciones, por ejemplo), como base para la innovación y la creatividad que permitan cautivar a los consumidores que se han vuelto más exigentes, informados y hedonistas.

Palabras clave: Gastronomía mexicana, tendencias de la gastronomía, consumo gastronómico, gastronomía tradicional.

*Correos electrónicos: alopezoj@uaemex.mx, culturayciudadania@gmail.com, cgh131060@yahoo.com.mx, riherlo@hotmail.com

TeORIA y PraXis · ISSN 18701582 · nÚM. 21 · ENERO-ABRIL 2017 · pp. 91-113 


\title{
Characterization of the main trends of Mexican gastronomy in the framework of new social scenarios
}

\author{
Andrés López Ojeda* \\ Universidad Autónoma del Estado de México \\ Carmen Pérez Camacho \\ C2 Cultura y Ciudadanía A. C. \\ Celia Guzmán Hernández \\ Ricardo Hernández López*Universidad \\ Autónoma del Estado de México
}

\begin{abstract}
Gastronomy in Mexico has consolidated as an important area for development and has come to the international forefront contributing to the strengthening of social relations. Traditional and popular gastronomy is a cultural identity factor. In this context, one of the objectives of this work consists in analyzing the main gastronomical trends which are characterized by their heterogeneity, but also by the fact of being part of larger processes, notably globalization, aestheticism of the offer and the emergence of new lifestyles and consumption patterns. Therefore, it is necessary to strengthen the knowledge of the gastronomy industry to understand its development and projection, particularly the field of professionalization and research considered as indispensable, especially in relation to greater knowledge of the own cuisines (for example reassessment, visibility, preservation of traditions) as a basis for innovation and creativity which allows to "seduce" new consumers who have become more demanding, informed and hedonistic.
\end{abstract}

KEY worDs: Mexican cuisine, gastronomy trends, gastronomic consumption, traditional cuisine.

*E-mails: alopezoj@uaemex.mx·culturayciudadania@gmail.com cgh131060@yahoo.com.mx·riherlo@hotmail.com 


\section{Introducción}

En los últimos años, el sector de la gastronomía en México se ha consolidado, como lo prueba el hecho de que, desde el punto de vista económico, aporta $2 \%$ del producto interno bruto y contribuye a generar cinco millones y medio de empleos directos e indirectos (Instituto Nacional de Estadística y Geografía, 2013). Pero eso no es todo, en el ámbito educativo, 354 instituciones imparten gastronomía en nivel superior y posgrado a 53412 alumnos (López Ojeda, Favila Cisneros y Vázquez Valdés, 2015). Todavía más: a partir de 2010, el interés global por la cocina mexicana se ha acentuado debido al reconocimiento de la cocina michoacana como patrimonio intangible de la humanidad y, como se puede constatar, por las búsquedas realizadas a través de Google, entre las que destacan las bebidas, productos y culinaria tradicionales.

Esta coyuntura ha propiciado que la gastronomía se haya constituido en un asunto de política pública al punto de que el gobierno federal comenzó a implementar, desde agosto de 2015, la Política de Fomento a la Gastronomía Nacional (PFGN), mediante la cual se pretende elevar la productividad del sector gastronómico en beneficio de los actores involucrados: productores, cocineros, emprendedores, así como de regiones y destinos de tradición, riqueza y diversidad gastronómica; es decir, de los diferentes eslabones y agentes que configuran la cadena de valor. Cabe señalar que la implementación de la PFGn ha quedado a cargo de la Secretaría de Turismo, lo cual se puede interpretar, en la agenda pública, como una prioridad el binomio turismo-gastronomía.

Debido al contexto sociocultural y económico de los últimos tiempos, se pueden identificar diversas tendencias gastronómicas que, por una parte, confirman la buena salud del sector y, por la otra, requieren su caracterización en términos de su pertinencia, alcances, limitaciones y aportes al conjunto social así como al complejo denominado gastronomía mexicana. El balance crítico de este panorama constituye el objetivo del trabajo, el cual tiene como fundamento una investigación documental y de campo realizada desde la perspectiva de los estudios de consumo y de mercado. Entre las tendencias que se analizarán se encuentran las siguientes: la gastronomía basada en el cosmopolitismo, las cocinas tradicionales y populares, la gastronomía turística patrimonialista, la cocina de autor, la estetización de la gastronomía, la gastronomía responsable, 
la gastronomía saludable y, para el caso del aspecto del consumo, los denomindados foodies.

Algunas de las conclusiones son las siguientes: la gastronomía en México es un asunto serio debido a su impacto en distintas dimensiones: cultural, social, económica, educativa, política e incluso identitaria; se ha conformado como un factor de desarrollo, y un caso particular lo reviste la órbita turística; la cocina tradicional y popular ha acrecentado su importancia y visibilidad en el mercado gastronómico; resulta necesario fortalecer el conocimiento del sector gastronómico para entender su desarrollo y proyección, particularmente el campo de la profesionalización y actualización que se vislumbra como imprescindible, sobre todo en lo referente al conocimiento de las propias gastronomías (revaloración, visibilidad, conservación de tradiciones, por ejemplo), así como para la innovación y creatividad que permitan "seducir" a los consumidores que se han vuelto más exigentes, informados y hedonistas.

\section{Política pública para la gastronomía}

El campo de la gastronomía en México se ha afirmado como una temática de interés por varias razones: propicia formas de identificación y relación social (la comida de familia, la comida con los amigos), genera elementos de identidad y distinción cultural (la comida indígena, la comida de fiesta, la cocina mexicana, la comida gourmet), así como toda una "economía del gusto" reflejada en el sector de la restauración y la industria alimenticia. Asimismo, la alimentación generalmente ha constituido un tema de política pública manifestado en la preocupación del Estado por atender problemas de desnutrición y hambre que amplios sectores de la población padecen todavía en México, además de mejorar las prácticas, consumos y hábitos alimenticios de las personas, como se puede apreciar en el caso de la llamada Cruzada Nacional contra el Hambre mediante el Programa Nacional México sin Hambre (http://sinhambre.gob.mx) o, para el caso de la capital del país, del programa SaludArte, que vincula salud nutricional, arte y educación, con un objetivo:

Busca que los niños y las niñas tomen conciencia de la importancia de comer sanamente a través de la formación en nutrición (Taller de nutrición), donde se incluyan a sus madres y padres (pláticas y demostraciones pedagógicas), con el respaldo 
de una asistencia alimentaria integral, para desarrollar habilidades en las y los niños que les permitan distinguir las características de una alimentación saludable [http://www.educacion.df.gob.mx/SaludArte/EducNutricional.php].

Por ello, resulta sumamente importante la PFGN, publicada el 5 de agosto de 2015 en el Diario Oficial de la Federación, la cual se puede leer como el reconocimiento a un campo creativo de los más significativos en este momento, no solo por su incidencia en términos de empleo, producción, cultura y turismo sino, sobre todo, como un eje de desarrollo. Al respecto, tal política se propone generar programas y acciones encaminadas a fortalecer la cadena de valor de la gastronomía considerando diferentes dimensiones: fomentar el desarrollo económico de las distintas gastronomías del país, usar de manera sustentable los recursos gastronómicos, fortalecer la dieta y el sistema alimentario mexicanos, proyectar la gastronomía en los niveles nacional e internacional, y desarrollar el conocimiento e innovación del sector, lo cual implicará, para el caso de la formación y capacitación:

1) Desarrollar redes de conocimiento especializado en temas de gastronomía mexicana.

2) Impulsar la investigación, innovación y transferencia tecnológica que contribuyan al desarrollo productivo de la gastronomía mexicana.

3) Impulsar la innovación y creatividad en la generación de nuevos productos y platillos que engrandezcan la cocina tradicional mexicana.

4) Asegurar la inclusión de temas tales como calidad en el servicio, inocuidad de los alimentos y gestión empresarial en escuelas gastronómicas.

5) Crear incentivos para el estudio y la innovación gastronómica en los centros de estudio nacionales y locales (PFGN, 2015, p. 14).

Cabe destacar que esa política que tiene en cuenta todos los eslabones de la cadena gastronómica será operada por la Secretaría de Turismo, lo cual le otorga una determinada orientación. Por el momento, la vinculación con el turismo es clara y puede contribuir dimensionando la importancia del campo gastronómico, pues si bien por lo regular ha ocupado un lugar secundario en relación con la diversidad, exuberancia y parafernalia de productos turísticos que se le ofrecen al visitante, actualmente se ha consolidado como un atractivo capaz de motivar los viajes de las personas; en este sentido, existen varios casos de 
éxito en España (Sayadi, 2001; Calvo, 2011; Ruiz Vega y Pelegrín Borondo, 2012) que demuestran la trascendecia de la gastronomía turística o el agroturismo. ¿A qué responde esta presencia de la gastronomía que ha generado un campo de conocimiento relevante para diversas disciplinas (medicina, nutrición, economía, administración, antropología, sociología, entre otras), sectores sociales, económicos e institucionales (organizaciones, ciudadanas, educativas, gubernamentales, empresariales), así como para el ciudadano promedio, sobre todo en su papel de consumidor de la oferta que se le brinda?.

Desde nuestro punto de vista, la importancia actual de lo gastronómico está vinculada con el llamado proceso de globalización, que ha impactado en distintos niveles y dimensiones, y en la manera en que se delinea y desarrolla tal fenómeno. Si bien lo que caracteriza a la globalización es el salto cualitativo en la dimensión económica y política en donde se ha tendido a una mayor articulación entre países a través de la integración de mercados (Mercado Común Europeo, Mercado Común del Sur), la aceleración de los intercambios de mercancías y bienes (Tratados de Libre Comercio de América del Norte), así como del sistema financiero internacional y la aparición de entidades supranacionales con una influencia cada vez mayor (Fondo Monetario Internacional, Banco Mundial), se pueden identificar otros procesos que inciden directamente en la forma en que se configuran diversas y actuales tendencias gastronómicas, las cuales se revisarán en los siguientes apartados.

\section{Tendencias actuales en la gastronomía mexicana}

\section{La gastronomía basada en el cosmopolitismo}

La intensificación de contactos interculturales como consecuencia del desarrollo de las comunicaciones convencionales (avión, automóvil, ferrocarril), de los llamados mass media (televisión, radio, cine pero, sobre todo, internet) y de la acentuación de la migración intranacional e internacional ha propiciado una conciencia de contemporaneidad (Appadurai, 1996); es decir, la percepción de que vivimos en un mundo más pequeño (compresión del tiempo-espacio) pero, también, el acceso y exposición a valores, símbolos, perspectivas, formas de pensar y visualizar el mundo que en otros momentos históricos no estaban tan rápidamente disponibles. En este aspecto, y para el caso de la gastronomía, es 
muy fácil identificar cómo los paisajes, principalmente urbanos, se han teñido de restaurantes y tiendas de la más diversa oferta cultural: en cualquier plaza comercial, por ejemplo, se puede encontrar comida china, japonesa, italiana, colombiana, argentina o brasileña. En la capital del país, las colonias Roma y Condesa han sido declaradas oficialmente, desde 2013, como un clúster creativo caracterizado por que en tal área existen más de 175 empresas dedicadas a diferentes actividades de la llamada economía creativa, y sobresalen los restaurantes de oferta gastronómica multicultural (INCUBARTE, 2015). Este proceso podemos sintetizarlo como una tendencia gastronómica basada en el cosmopolitismo.

\section{Cocinas tradicionales y populares}

La aceleración, producción y monopolio de los flujos de información y datos, como consecuencia de las novedosas tecnologías de comunicación, no solo se constituyen en la base de la economía actual, sino también han comenzado a plantear la transición hacia un nuevo paradigma social fundamentado en el conocimiento (Castells, 1996). Al respecto, el texto Hacia las sociedades del conocimiento. Informe mundial de la Organización de las Naciones Unidas para la Educación, la Ciencia y la Cultura (Unesco, 2005) consigna que, si bien los montos de información son importantes en términos de, por ejemplo, acceso y difusión de las innovaciones y aumentos de la productividad, el "vasto potencial cognitivo" con el que cuentan los diversos pueblos del mundo es trascendental en la medida en que constituye un conocimiento que crea sentido, por lo que conviene valorizarlo.

Lo anterior resulta cardinal en términos de que, frente al desbordamiento de la información dadas las nuevas posibilidades de la internet y los medios audiovisuales, así como debido al fortalecimiento de la hegemonía cultural angloparlante y de lo occidental, el lugar que ocupan los conocimientos locales y la diferencia cultural se legitima, como se enfatiza en el mismo informe: "esta perspectiva no obedece exclusivamente a un imperativo abstracto de carácter ético, sino que apunta principalmente a suscitar en cada sociedad una toma de conciencia de la riqueza de los conocimientos y capacidades de que es depositaria a fin de que los valore y aproveche mejor” (Unesco, 2005, p. 18). En el terreno cultural, ello explica la revalorización de la diferencia cultural, de lo local 
frente a lo global, lo cual se conecta, en el plano gastronómico, por la tendencia a visibilizar las cocinas tradicionales y populares. En este sentido, los chefs latinoamericanos más reconocidos y sus restaurantes, cada vez mejor situados en las más prestigiosas listas gastronómicas (San Pellegrino, Restaurant), han fundado sus propuestas en sus cocinas nacionales convirtiéndose al mismo tiempo en promotores de las mismas, como el caso de Enrique Olvera para la cocina tradicional mexicana, Virgilio Martínez para la cocina incaica, y Alex Atala, quien en su propuesta "privilegia ingredientes genuinamente brasileños" (http://domrestaurante.com.br/pt-br/sabor.html).

\section{Gastronomía turística patrimonialista}

Lo anterior se encuentra vinculado con la revalorización del patrimonio no solo como bien simbólico que incide en la construcción de nacionalismo y pertenencia comunitaria, sino también como factor de desarrollo. En el primer caso, la inscripción de una de las cocinas tradicionales de México a la Lista Representativa del Patrimonio Cultural Inmaterial de la Humanidad -el "Paradigma Michoacano" (Unesco, 2015) - refuerza el interés por las propias gastronomías frente a los anteriores modelos predominantes que constituyeron la medida de lo refinado, lo culto o lo sofisticado, por ejemplo las cocinas francesa o italiana, las cuales se siguen impartiendo en la mayoría de los programas gastronómicos educativos como modelos de la "alta cocina". En México solo existe un programa de posgrado en Gastronomía orientado a apoyar el trabajo de promoción y rescate del programa de salvaguarda que implica tal reconocimiento (se trata de la maestría en Cocinas de México, impartida por la Culinary Art School en Tijuana, Baja California). En el segundo caso -sobre todo relacionado con el turismo-, las gastronomías ancestrales, tradicionales y locales han comenzado a ocupar un lugar principal al punto de que existe un programa de nivel federal centrado en ellas (véase cuadro 1), el cual recupera diversos valores asociados con la gastronomía como recurso: su valor económico, histórico y cultural. Es decir, hay una tendencia por la gastronomía turística patrimonialista. 
CuAdro 1. Rutas turístico-gastronómicas

\begin{tabular}{|c|c|c|}
\hline Ruta & Gastronomía/producto & Estado(s) \\
\hline $\begin{array}{l}\text { Cocina de Dos } \\
\text { Mundos }\end{array}$ & Cocina rarárumi & Chihuahua \\
\hline Del Café a la Vainilla & Vainilla & Veracruz \\
\hline Del Mar a la Laguna & $\begin{array}{l}\text { Cocina del mar y } \\
\text { trópico }\end{array}$ & Tamaulipas \\
\hline $\begin{array}{l}\text { El Altar de Día de } \\
\text { Muertos }\end{array}$ & $\begin{array}{l}\text { Cocina michoacana } \\
\text { (dulces, carnitas) }\end{array}$ & Michoacán \\
\hline El Sabor de Hoy & $\begin{array}{l}\text { Cocina fusión y de } \\
\text { vanguardia }\end{array}$ & Distrito Federal \\
\hline El Sazón del Minero & Cocina virreinal & Aguascalientes, San Luis Potosí \\
\hline Ruta & Gastronomía/productos & Estado(s) \\
\hline $\begin{array}{l}\text { Entre Cortes y } \\
\text { Viñedos }\end{array}$ & Productos cárnicos & $\begin{array}{l}\text { Coahuila, Nuevo León, } \\
\text { Durango, Sonora }\end{array}$ \\
\hline $\begin{array}{l}\text { La Cocina al Son del } \\
\text { Mariachi }\end{array}$ & Pozole, birria, tequila & Jalisco \\
\hline $\begin{array}{l}\text { La Cultura del } \\
\text { Maguey }\end{array}$ & Barbacoa, pulque & Hidalgo \\
\hline $\begin{array}{l}\text { La Mesa de la Huerta } \\
\text { y el Mar }\end{array}$ & Cocina del mar & Sinaloa \\
\hline La Ruta del Cacao & Cocina chiapaneca & Chiapas \\
\hline $\begin{array}{l}\text { Los Dulces Sabores de } \\
\text { Antaño }\end{array}$ & Dulces tradicionales & Tlaxcala, Puebla \\
\hline $\begin{array}{l}\text { Los Fogones entre } \\
\text { Viñas y Aromas del } \\
\text { Mar }\end{array}$ & Cocina del mar, vino & $\begin{array}{l}\text { Baja California Norte y Baja } \\
\text { California Sur }\end{array}$ \\
\hline $\begin{array}{l}\text { Los Ingredientes } \\
\text { Mestizos del Mayab }\end{array}$ & $\begin{array}{l}\text { Cocina del mar y tierra: } \\
\text { miel, chile habanero, } \\
\text { achiote }\end{array}$ & $\begin{array}{l}\text { Campeche, Yucatán, Quintana } \\
\text { Roo }\end{array}$ \\
\hline Los Sabores del Mar & Cocina del mar & Colima, Nayarit \\
\hline Platillos con Historia & Cocina regional & Querétaro y Guanajuato \\
\hline Ruta de los Mercados & $\begin{array}{l}\text { Productos e } \\
\text { ingredientes regionales }\end{array}$ & Estado de México, Guerrero \\
\hline
\end{tabular}

Fuente: Atlas Turístico de México (http://atlasturistico.sectur.gob.mx/AtlasTuristico/inicio. do?lang $=$ ES\#). 


\section{Cocina de autor}

Continuando con la relevancia del conocimiento en las economías postindustriales, también se aprecia cómo las llamadas industrias creativas representan un sector cada vez más importante, pues además de que contribuyen al crecimiento económico y a la creación de empleo, actúan como elementos vehiculares en la transmisión de la identidad cultural, aspecto esencial en la difusión y promoción de la diversidad cultural. Una definición señala: "El término industria creativa supone un conjunto más amplio de actividades que incluye a las industrias culturales más toda producción artística o cultural, ya sean espectáculos o bienes producidos individualmente. Las industrias creativas son aquellas en las que el producto o servicio contiene un elemento artístico o creativo substancial e incluye sectores como la arquitectura y publicidad" (Unesco, s. f., p. 2). En el campo de la gastronomía se advierte cómo, para la dimensión de la oferta y el consumo, la creatividad e innovación son componentes fundamentales que explican la aparición de nuevos servicios y productos donde se despliegan, sobre todo, los componentes creativos e innovadores individuales, como en el caso de la tendencia a la cocina de autor. Al respecto, Cartay (2015, s. p.) comenta que:

El término de cocina de autor es relativamente nuevo. Uno de sus impulsores iniciales fue el detective Pepe Carvalho, un personaje de Manuel Vázquez Montalbán, entusiasta de la cocina, quien señaló en un parlamento de novela que, con la cocina de autor, el cocinero expresa su madurez creativa, superando la dictadura del chef que se adapta al establishment. Con la cocina de autor, el cocinero crea un estilo propio con una propuesta singular [...] La cocina de autor mezcla, en una simbiosis creativa, los principios de la cocina tradicional con los de la Nouvelle Cuisine y la Cocina de Fusión. Toma lo mejor de los otros y lo presenta a su manera, en una propuesta creativa, marcada por su sello personal. Como en muchos casos, el chef es dueño parcial o total del restaurante, debe saber desenvolverse en muchas disciplinas o temas: cocina, higiene, nutrición, dietética, administración, etcétera. Generalmente ofrece un menú de degustación, escogiendo sus mejores creaciones para presentar al cliente un menú representativo de sus creaciones en pequeñas y estudiadas porciones. 


\section{Estetización de la gastronomía}

En el terreno nuevamente de la gastronomía como factor de desarrollo existe otro proceso de naturaleza histórica que va delineando lo que llamaremos tendencia a la estetización de la gastronomía. Gilles Lipovetsky y Jean Serroy (2015) indican que nos encontramos en una época caracterizada por el "esteticismo". Como jamás ha sucedido en la historia, el consumo ha comenzado a entronizarse y asistimos a la etapa del capitalismo del hiperconsumo que ha logrado articular la esfera productiva y la cultural. En específico, la producción, distribución y consumo están impregnados por operaciones de naturaleza fundamentalmente estética. Esto quiere decir que hay que abrir o reconocer otra dimensión al consumo: no solo propiamente económica de compra compulsiva de mercancías (consumismo), de distinción y diferencia social (Bourdieu), o como un ejercicio de la ciudadanía y los derechos (García Canclini), sino también como parte de un modo de producción estético (Lipovetsky y Serroy). Esto explica, por ejemplo, que aun cuando las personas sean conscientes de que una compra no es "racional" porque se pueden arruinar o endeudar casi para toda la vida, la realicen "por su diseño", "porque es bonito" o "por su color".

En el caso de la gastronomía, existen hasta cursos para convertir las comidas en un performance. Poco a poco va quedando atrás la glorificación de los chefs y de los platillos que salen de las cocinas impecables y listos para inmortalizarse en el smartphone. Lo más novedoso son las técnicas de emplatado a la vista del cliente, el servicio centrado en el producto para generar un espectáculo, es decir, la escenificación de la alimentación. En este tenor, resulta ilustrativo de esta tendencia los cursos que se imparten en el Basque Culinary Center, una de las instituciones de vanguardia en el campo gastronómico, en donde se aprecian claramente los aspectos sensibles y estéticos como valores agregados (cuadro 2).

Lo que se quiere enfatizar es que, en la industria creativa de la restauración, se ha comenzado a dejar atrás la mera visión de los alimentos en términos de su aporte funcional (a la nutrición, a la salud) para convertirlos en un objeto de deseo, como una manifestación de la sensibilidad y un artículo estético en sintonía con el proceso de estetización actual. 
Cuadro 2. Talleres impartidos en el Basque Culinary Center para incrementar la experiencia visual del comensal

\begin{tabular}{|c|c|}
\hline Nombre del taller & Propósito \\
\hline $\begin{array}{l}\text { Elaboración de una carta y } \\
\text { otros elementos gráficos }\end{array}$ & $\begin{array}{l}\text { Entender la importancia de los elementos gráficos } \\
\text { como parte de la imagen del establecimiento y } \\
\text { herramienta de venta. }\end{array}$ \\
\hline $\begin{array}{l}\text { Análisis sensorial de la } \\
\text { cubertería }\end{array}$ & $\begin{array}{l}\text { Se crea conciencia de la importancia del material } \\
\text { del cubierto a la hora de comer. Viviremos } \\
\text { una experiencia sensorial utilizando diferentes } \\
\text { materiales. }\end{array}$ \\
\hline $\begin{array}{l}\text { Corporalidad. El lenguaje no } \\
\text { verbal }\end{array}$ & $\begin{array}{l}\text { Se aprende a gestionar movimientos en la sala } \\
\text { tanto individualmente como en equipo. Afinar el } \\
\text { cuerpo que respira, que se mueve, que habla, que } \\
\text { piensa, siempre en armonía con el espacio y los } \\
\text { otros, creando belleza. }\end{array}$ \\
\hline
\end{tabular}

\section{Gastronomía saludable}

No obstante la tendencia anterior, y ante el reto que impone la consecución de la sostenibilidad como contrapeso a las consecuencias negativas de la globalización y el predominio del hipercapitalismo, que también, es cierto, repercute en la sobreexplotación de los recursos patrimoniales -ya sea naturales (cambio climático) o culturales (homogeneización de valores y patrones de consumo)-, constantemente se plantea la pregunta de cómo se va conseguir la sustentabilidad del modelo actual de desarrollo. En el campo del turismo gastronómico ello implica, según las conclusiones del I Foro Mundial de Turismo Gastronómico, celebrado en abril de 2015 en San Sebastián, España, considerarla a partir de tres pilares: ambiental (reduciendo las emisiones), sociocultural (la autenticidad del destino) y económica (que se distribuya de manera equitativa). El turismo gastronómico -se enfatizó- puede ser todo menos de masas y debe ser ético con el entorno para que perdure. Esto se enlaza, por ejemplo, con la tendencia actual del agroturismo caracterizado por centrarse en el desarrollo local, participativo, solidario, y con la preocupación por diseñar productos agroalimentarios que sean amables con los entorno -sobre todo rurales-, elaborados de manera artesanal y orgánicamente con base en el "saber hacer" tradicional. Al respecto, el Conservatorio de la Cultura Gastronómica de México, entidad que formuló el expediente para el reconocimiento del paradigma gastronómico michoacano 
como patrimonio intangible, cuenta con un programa orientado a revalorar el papel de las cocineras tradicionales que resulta coherente con esas tres dimensiones de la sustentabilidad: promueve el reconocimiento de la autenticidad de una cocina local producida de manera racional a partir de los saberes tradicionales, en un contexto de autenticidad cultural y con equidad de género.

Otra dimensión de la sostenibilidad para el campo gastronómico tiene que ver con la necesidad de producir más "naturalmente" en oposición al modelo predominante de la industria alimenticia caracterizada por la utilización de fertilizantes, agroquímicos, y la alteración de los procesos (a partir de los hallazgos de la biotecnología). Al respecto, existe una abundante literatura que se acerca a la temática de manera ambivalente debido a que, por una parte, la inversión en tecnología ha ocasionado que, por ejemplo, aumente la vida de anaquel de los alimentos; el uso de empaques y otros medios de contención facilita su manipulación y traslado permitiendo la posibilidad de grandes volúmenes de distribución y venta; asimismo, el procesamiento de los alimentos ha permitido la modificación de sabores y características para mejorar su palatabilidad e incluso incrementar su valor nutricional que podría influir de manera positiva en el estado nutricional de la población. Pero, por otra parte, debido a la composición y química del procesamiento de los alimentos industrializados (por ejemplo, niveles aumentados de sodio y bajo contenido de fibra), existe preocupación por sus posibles efectos en la salud de las personas (González et al., 2007).

Lo anterior es relevante, en especial porque el consumo de alimentos industrializados alcanzó, en 2013, los 137 millones de dólares y se prevé un crecimiento anual de 6.2 \% hasta el año 2020 (ProMéxico, 2014, p. 17). En el mismo sentido, García (2012, p. 196) menciona que las ciudades de México, Mérida y Tijuana tienen alta preferencia por los alimentos procesados y, en específico, la mitad de amas de casa de cinco ciudades (Ciudad de México, Monterrey, Guadalajara, Tijuana y Mérida) y los jóvenes (55 \%). Por niveles socioeconómicos, los de altos ingresos son quienes mayoritariamente consumen alimentos procesados y, a medida que baja el nivel de ingresos, desciende la preferencia hasta $20 \%$ menos respecto al nivel más bajo.

Según el estudio de García (2012, p. 197), si bien los alimentos procesados y/o enlatados se perciben mucho más prácticos, "existe una cierta imagen 
negativa en torno a la inseguridad y el valor nutritivo de los alimentos enlatados, pues se cree que contienen plomo, menos nutrientes, son menos frescos y tienen conservadores artificiales; por lo tanto se percibe como peligroso consumir alimentos en latas". Salvo lo mencionado, no hay estadísticas ni estudios que nos presenten la situación de los consumos y prácticas alimenticias de este tipo de productos. Como sea, tales creencias han llevado a la emergencia de una tendencia que podemos denominar gastronomía saludable. Esto implica partir de ciertos principios para el consumo de alimentos: moderación, variedad, equilibrio, así como la "aplicación de métodos y técnicas (arte) en la preparación de alimentos con el propósito de conservar sus moléculas y valores nutricionales, además de asegurar el atractivo en términos de color, sabor, textura y apariencia” (Caldera, 2010, s. p.). Lo que implica esta tendencia es el paso de la fast food a la fast good. Un ejemplo lo constituye la cadena española Summac Foods, que en pleno centro financiero de Madrid y rodeado de empresas y oficinas se enfoca básicamente a gente de negocios que no dispone de mucho tiempo para comer pero que desea hacerlo de forma sana. Un estilo similar fue el que inspiró a David Vázquez, del Mercado Habif, para abrir en la ciudad de Metepec Salad \& Salad, Ensaladas a tu Gusto, donde lo primero que encuentra el consumidor es una barra con más de 25 ingredientes para preparar las ensaladas así como vinagretas y aderezos artesanales, es decir, sin conservadores (Castaños Rocha, 2015).

El punto es importante porque, de acuerdo con la Secretaría de Agricultura, Ganadería, Desarrollo Rural, Pesca y Alimentación (Sagarpa, 2013), la demanda de alimentos orgánicos certificados en México registra un incremento de $10 \%$ anual, y existen 169500 productores que trabajan superficies de cultivo orgánico que alcanzan las 351904 hectáreas con ventas que han aumentado $20 \%$ en las tiendas de autoservicio y $10 \%$ en tiendas especializadas. Si bien los productos orgánicos todavía resultan caros, cada vez son más frecuentes iniciativas como la que se desarrolla en la Ciudad de México donde se puede encontrar el llamado Mercadito 100, que intenta no solo acercar tales productos a los citadinos, sino además "promover una forma de vida más responsable con el medio ambiente y las personas, en una era impactada cada vez más por los malos hábitos de consumo urbano" a partir de cinco valores fundamentales para su oferta: que sea local, sana, rica, justa y limpia (Nájera y Corpi, 2013, s. p.). 
Lejos está, en este caso, la mera producción y comercialización de alimentos, pues también esta tendencia implica nuevas formas de relación, participación y educación en lo alimenticio. Así, por ejemplo, en el Mercadito 100 se desarrolla un Sistema de Garantía Participativa, donde el consumidor puede verificar la forma de producción, y se fomenta la cultura del comercio justo y del consumo responsable.

Hasta aquí, se puede decir que para caracterizar las tendencias en el campo de la gastronomía es preciso comprenderla como actividad económica, cultural, social y profesional que responde, sobre todo, a las demandas del mercado y a procesos de naturaleza estructural (globalización, estetización del capitalismo, sostenibilidad, turismo). En este contexto, una pregunta pertinente surge en torno a cuáles son las características de la generación de profesionales y mercado de consumo gastronómico que se puede perfilar en los próximos años.

\section{Foodies}

Una generación es un grupo social que comparte a lo largo de su historia un conjunto de experiencias formativas que los distinguen de sus predecesores. Estos conjuntos tienen por base principios sociohistóricos compartidos entre los que se pueden contabilizar, por ejemplo, la visión de vida, valores y símbolos. Hoy en día, conviven, comparten e interactúan en el mercado laboral y de consumo cuatro generaciones: los tradicionalistas (nacidos antes de 1945), los baby boomers (1946-1963), la Generación X (1964-1979) y la Generación Y (1980-2000) (González, 2011).

Esta última generación es la que egresa actualmente de las universidades, se incorpora al trabajo profesional y, en los próximos años, formará el grueso de los consumidores; asimismo, implica un reto metodológico para las empresas ya que, según Castañeda (2013a, 2013b), está poco inclinada a poseer objetos: casas, muebles, autos u objetos de valor suntuario. De acuerdo con el autor, son menos ávidos de bienes patrimoniales pues existe la idea de que la posesión representa sumisión e inmovilidad. A diferencia de sus padres, están más interesados en el presente, la libertad de acción, la socialización y el consumo cultural entendido como experiencia. 
Con base en la última reunión anual del Institute of Food Technology, las tendencias de los consumidores de la Generación Y alcanzarán los 64 millones tan solo para Estados Unidos. Lindsey (2014) reporta que este es un mercado informado respecto a la tecnología, la alimentación, y está preocupado en cómo impactan sus decisiones de consumo en el medio ambiente, lo cual resulta coherente con el marco de la globalización y los cambios que se han generado en torno a la constitución de sociedades de conocimiento, patrimonialización, etcétera, analizados anteriormente. Para el caso de la gastronomía, quienes pertenecen a la llamada Generación Y se interesan en experiencias que impliquen una conexión directa con lo que comen, con quien cocina y la personalización del producto final.

Distintos autores (Halperin, 2012; Hoffman, 2012; Barton, Koslow, Fromm y Egan, 2012) concuerdan en que esta generación posee cuatro características principales que delinearán el futuro mercado de consumo gastronómico, así como las necesidades de formación, producción y ofertas de nuevos productos para quienes conforman la ecología de tal campo:

a) Anhelo por la diversidad auténtica, es decir, pertenencia a la "aldea global” pero con plena conciencia de que también se coexiste con la diferencia cultural, además de una inclinación para viajar y atesorar experiencias que den cuenta de ello.

b) El convencimiento de que un tamaño definitivamente no ajusta a todos pues quieren tener decisión sobre lo que comen en el plano individual, buscan personalizar sus opciones de menú, son quisquillosos con aquello que se vincula con la producción industrial y representa un mercado heterogéneo.

c) Son conscientes de la salud aunque propensos a arrebatos decadentes, esto es, son cuidadosos con su forma física y están informados sobre nutrición, sin embargo, continúan consumiendo comidas hipercalóricas.

d) Argumentan una conciencia social alimentaria con tendencia a la comida orgánica, amigable con los derechos animales, cultivados localmente y libres de hormonas, entre otros compuestos químicos.

Este traslape entre el movimiento general del contexto sociocultural y las características de muchos de los actuales y futuros consumidores da pie a la 
llamada tendencia foodie. Según Ward (2013), los jóvenes que ahora se encuentran entre los 20 y 30 años han comenzado a sustituir los cafés y bares por los restaurantes como centros de socialización. De acuerdo con Tomás e Iglesias (2013), ser foodie va más allá de estar incluido en una simple tribu urbana 0 una nueva tendencia gastronómica: es una actitud hacia la comida, la cual construye un nuevo estilo de vida, un interés por articular las diferentes manifestaciones de la gastronomía en esa visión que comparten: desde una cata de vino, el hallazgo de una nueva panadería, hasta el interés por la ciencia de los alimentos.

Es un mercado de la gastronomía que demanda experiencias totalizadoras, que buscan en todo momento el saber que guarda el sabor, lo que ha propiciado un cambio en la oferta de productos y servicios en el amplio campo de la alimentación. Donde han proliferado revistas especializadas, secciones en los diarios, blogs y programas televisivos sobre comida y gastronomía, mercados de agricultores, tiendas de productos artesanales y locales, food trucks, festivales, ferias y exposiciones gastronómicas; incluso en el turismo gastronómico y rural resulta necesario fortalecer la formación y capacitación de quienes prestarán y ofrecerán servicios acordes a los nuevos escenarios.

\section{Retos de las tendencias para el campo profesional gastronómico}

La aparición de nuevas tendencias en la gastronomía forma parte de procesos estructurales de nivel global y nacional de orden económico, social y cultural que repercuten en la aparición de nuevos consumos y, para el caso del ámbito profesional, exigencias de formación y orientación de programas académicos viables y coherentes con tal marco. En este sentido, el cuadro 3 relaciona algunas de las tendencias gastronómicas actuales, su vinculación/determinación con el contexto que las impulsa y los retos disciplinarios que plantean, sobre todo en relación con la necesidad de crear programas de mayor nivel que den respuesta a las demandas. 


\section{Cuadro 3. Retos de formación para el campo gastronómico actual}

\begin{tabular}{|c|c|c|}
\hline Tendencia & $\begin{array}{l}\text { Influencia del contexto sociocultural } \\
\text { y económico }\end{array}$ & Retos de formación y capacitación \\
\hline $\begin{array}{l}\text { Gastronomía } \\
\text { basada en el } \\
\text { cosmopolitismo }\end{array}$ & $\begin{array}{l}\text { Proceso de globalización que } \\
\text { acentúa la exposición a tradiciones } \\
\text { gastronómicas diversas. }\end{array}$ & $\begin{array}{l}\text { Además de los conocimientos } \\
\text { disciplinarios, ampliación de } \\
\text { saberes relacionados con otras } \\
\text { tradiciones culinarias (historia, } \\
\text { valores, ingredientes, productos). }\end{array}$ \\
\hline $\begin{array}{l}\text { Visibilización } \\
\text { de las cocinas } \\
\text { tradicionales y } \\
\text { populares }\end{array}$ & $\begin{array}{l}\text { Reacción frente al proceso } \\
\text { globalizado de homogeneización } \\
\text { sociocultural. } \\
\text { Patrimonio y diferencia cultural } \\
\text { como valores deseables alentado por } \\
\text { instituciones internacionales como } \\
\text { la Unesco. }\end{array}$ & $\begin{array}{l}\text { Formación en conocimientos } \\
\text { disciplinarios de gastronomías } \\
\text { locales y profundización de la } \\
\text { cultura alimentaria propia. }\end{array}$ \\
\hline $\begin{array}{l}\text { Gastronomía } \\
\text { turística } \\
\text { patrimonialista }\end{array}$ & $\begin{array}{l}\text { Tendencia del turismo cultural a } \\
\text { la alza y mayor importancia de las } \\
\text { gastronomías locales como atractivo. }\end{array}$ & $\begin{array}{l}\text { Formación en conocimientos } \\
\text { disciplinarios de gastronomías } \\
\text { locales, profundización de la } \\
\text { cultura alimentaria propia y } \\
\text { creatividad para la innovación de } \\
\text { servicios y productos. }\end{array}$ \\
\hline Cocina de autor & $\begin{array}{l}\text { Emergencia de las sociedades del } \\
\text { conocimiento y alta valoración de la } \\
\text { creatividad y la innovación. Auge del } \\
\text { individualismo, de la diferenciación } \\
\text { o singularidad. }\end{array}$ & $\begin{array}{l}\text { Innovación y creatividad } \\
\text { de servicios y productos } \\
\text { gastronómicos diferenciados o } \\
\text { singulares. } \\
\text { Perfeccionamiento de técnicas } \\
\text { novedosas u originales. } \\
\text { Habilidades y conocimientos para } \\
\text { los nuevos emprendimientos. }\end{array}$ \\
\hline $\begin{array}{l}\text { Estetización de } \\
\text { la gastronomía }\end{array}$ & $\begin{array}{l}\text { Estadio del capitalismo como } \\
\text { modo de producción estético y de } \\
\text { hiperconsumo (Lipovetsky y Serroy). }\end{array}$ & $\begin{array}{l}\text { Ampliación de conocimientos } \\
\text { multidisciplinarios que aporten } \\
\text { a la generación de experiencias } \\
\text { estéticas a través de la } \\
\text { gastronomía. }\end{array}$ \\
\hline $\begin{array}{l}\text { Gastronomía } \\
\text { saludable }\end{array}$ & $\begin{array}{l}\text { Respuesta al tema de la } \\
\text { sustentabilidad, los movimientos } \\
\text { enfocados a la conservación de los } \\
\text { recursos naturales, aparición de } \\
\text { problemas de salud derivados de } \\
\text { la mala alimentación (slow food, } \\
\text { gastronomía lógica). }\end{array}$ & $\begin{array}{l}\text { Conocimientos disciplinarios y } \\
\text { funcionales de los alimentos, } \\
\text { sobre todo de aquellos derivados } \\
\text { de la gastronomía tradicional y } \\
\text { popular. }\end{array}$ \\
\hline $\begin{array}{l}\text { Tendencia } \\
\text { foodie }\end{array}$ & $\begin{array}{l}\text { Perfil de consumidores más } \\
\text { exigentes, mayormente informados, } \\
\text { hedonistas, que les gusta } \\
\text { diferenciarse. }\end{array}$ & $\begin{array}{l}\text { Aspectos mercadológicos, de } \\
\text { innovación y creatividad para } \\
\text { crear ambientes para los nuevos } \\
\text { consumidores. }\end{array}$ \\
\hline
\end{tabular}

Fuente: Elaboración propia (2015). 


\section{Conclusiones}

Recientemente se anunció que se alista un decreto presidencial para declarar el 16 de noviembre como Día Nacional de la Gastronomía en México, lo cual confirma la importancia del sector. Todavía más: según la propuesta, las secretarías de Cultura y de Turismo "realizarán las acciones necesarias para concretar la instrumentación de dicha conmemoración” (Téllez Cortés, 2016, p. 6). La noticia es paradigmática del nivel alcanzado por un sector que se vincula con 150 de las 303 ramas clasificadas en el Sistema de Clasificación Industrial de América del Norte (Cámara Nacional de la Industria de Restaurantes y Alimentos Condimentados, 2015), lo cual ilustra las intrincadas ramificaciones que puede alcanzar esa entidad denominada gastronomía mexicana.

En términos de la oferta y el consumo, se puede prever un saludable dinamismo habida cuenta, por una parte, las nuevas demandas explicadas por procesos amplios como la globalización, los cambios alimenticios, el estilo de vida saludable y sustentable que motivarán respuestas innovadoras desde el campo gastronómico y, por la otra, la aparición de nuevas clases de gastrósofos altamente demandantes y ávidos de productos con mayor valor agregado y claramente diferenciados.

Por el momento, el texto presenta un primer acercamiento a las configuraciones actuales en el panorama de la gastronomía mexicana entre las que resalta una tendencia a la revaloración de la cocina tradicional donde se aprecian dos actores principales: $a$ ) los chefs de alta cocina que han volteado a ver la propia tradición gastronómica incorporando a sus propuestas ingredientes, productos, técnicas y saberes locales; $b$ ) la institucionalidad pública a través de una política de fomento que intenta incidir en toda la cadena de valor del sector gastronómico; si bien la estrategia ostenta un alto componente utilitario (la implementación de la política en manos de la Secretaría de Turismo), también intenta fortalecer a la gastronomía como un elemento de identificación cultural.

Otra consideración relevante implica el hecho de visualizar a la gastronomía como un elemento de desarrollo por su aporte a la generación de empleo e ingreso que explica, por ejemplo, la articulación de la política de fomento a la órbita del turismo. Un reto, en este sentido, sería la creación de productos gastronómicos capaces de complacer a los distintos consumidores. La gastronomía nacional, en este terreno, tiene un área de oportunidad toda vez que, en 
México, el turista nacional exhibe un interés claro por las cocinas regionales.

Por último, cabe señalar que la construcción de la oferta y la satisfaccion del consumo gastronómico, que se puede visualizar como un colorido mosaico, debe considerar como una clave a la profesionalización y la investigación que se vislumbra como imprescindible, sobre todo en lo referente al mayor conocimiento de las propias gastronomías (revaloración, visibilidad, conservación de tradiciones, por ejemplo), pues ello servirá para impulsar la innovación y la creatividad que permitan "seducir" a los actuales consumidores que, como se mencionó, ahora son más exigentes, informados y hedonistas.

\section{Fuentes consultadas}

Appadurai, A. (1996). Modernity at Large: Cultural Dimensions of Globalization. Mineápolis: University of Minnesota Press.

Barton, C., Koslow, L., Fromm, J. y Egan, C. (2012). Millennial Passions: food, fashion, and friends. bcg.perspectives. Recuperado de http://www.bcg. com/documents/file121010.pdf

Caldera, Y. (2010). Gastronomía saludable. ¿Se puede comer sano y sabroso? Recuperado de http://es.slideshare.net/calidadyalimentum/gastronomiasaludable

Calvo, D. (2011). Productos agroalimentarios de calidad, gastronomía y patrimonio cultural: activos para la promoción del territorio y de destinos turísticos. En C. Fabián y C. Fandos (coords.), Turismo gastronómico. Estrategias de marketing y experiencias de éxito (pp. 93-116). Zaragoza: Prensas Universitarias de Zaragoza.

Cámara Nacional de la Industria de Restaurantes y Alimentos Condimentados. (2015). Todo sobre la mesa. Dimensiones de la industria restaurantera. México: Autor.

Cartay, R. (2015). Todo sobre la cocina de autor. Cocina y Vino. Recuperado de http://cocinayvino.net/gastronomia/especiales/1803-todo-sobrela-cocina-de-autor.html 
Castañeda, A. (2013a). Los Millennials: ¿Es que los chavos no desean poseer autos 0... nada? Merca2.0. Recuperado de http://www.merca20.com/ los-millennials-es-que-los-chavos-no-desean-poseer-autos-o-nada

Castañeda, A. (2013b). Los Millennials: Trabajo, Casa y Relación. Merca2.0. Recuperado de http://www.merca20.com/los-millennials-trabajo-casa-yrelacion

Castaños Rocha, N. (2015). Abre un negocio de cocina saludable. Recuperado de http://www.soyentrepreneur.com/5012-abre-un-negocio-de-comida-saludable.html

Castells, M. (1996). La era de la información. Madrid: Alianza.

García, P. (2012). La alimentación de los mexicanos. Cambios sociales y económicos, y su impacto en los hábitos alimenticios. México: Cámara Nacional de la Industria de Transformación.

González, R. (2011). La incorporación de la Generación Y al mercado laboral. Palermo Business Review, 5, 67-93.

González, D. et al. (septiembre-octubre, 2007). Alimentos industrializados en la dieta de los preescolares mexicanos. Revista Salud Pública de México, 49(5), 345-356.

Halperin, M. (marzo, 2012). Fulfilling Generation Next. QSRmagazine. Recuperado de http://www.qsrmagazine.com/marc-halperin/fulfilling-generation-next

Hoffman, B. (2012). How "Millennials" are Changing Food as we Know it. Forbes. Recuperado de http://www.forbes.com/sites/bethhoffman/2012/09/04/how-millenials-are-changing-food-as-we-know-it

INCUBARTE. (2015). En la Roma-Condesa DF se oficializa un cluster creativo. Recuperado de http://incubarte.mx/blog/en-la-roma-condesa-df-seoficializa-un-cluster-creativo/

Instituto Nacional de Estadística y Geografía. (2013) Cuenta satélite del turismo de México, 2013. México: Autor.

Lindsey, K. (24 de junio de 2014). Food Trends: Get to know tne millennial generation. Food Dive. Recuperado de http://www.fooddive.com/news/ food-trends-get-to-know-the-millennial-generation/278390

Lipovetsky, G. y Serroy, J. (2015). La estetización del mundo. Vivir en la época del capitalismo artístico. Barcelona: Anagrama. 
López, A. Favila H. y Vázquez T. (2015). Mapeo de la oferta profesional en gastronomía a nivel nacional. Culinaria Revista Virtual Especializada en Gastronomía, 10, 31-45.

López Ojeda, A., Favila Cisneros, H. y Vázquez Valdés, T. (julio-diciembre, 2015). Mapeo de la oferta profesional en gastronomía a nivel nacional. Culinaria. Revista Virtual Especializada en Gastronomía.

Nájera, A. y Corpi, S. (20 de septiembre de 2013). El nuevo mercadito mexicano. El mercado se reinventa (DF y GDL). Recuperado de http://www. reporteindigo.com/el-wiken/breik/el-mercado-se-reinventa-df-y-gdl

Política de Fomento a la Gastronomía Nacional 2014-2018 (2015). Disponible en http://www.cultura.gob.mx/turismocultural/documentos/pdf/Politica_de_fomento_a_la_gastronomia_nacional.pdf

ProMéxico. (2014). Alimentos procesados. México: Secretaría de Economía.

Ruiz Vega, A. V. y Pelegrín Borondo, J. (2012). Estrategias empresariales seguidas en turismo enológico: análisis de casos prácticos en España. En C. Flavián y C. Fandos (coords.), Turismo gastronómico. Estrategias de marketing y experiencias de éxito (pp. 119-190). Zaragoza: Prensas Universitarias de Zaragoza.

Sagarpa (Secretaría de Agricultura, Ganadería, Desarrollo Rural, Pesca y Alimentación) (13 de abril de 2013). Fomenta SAGARPA producción y consumo de alimentos orgánicos en México y el mundo. Recuperado de http://www.sagarpa.gob.mx/saladeprensa/2012/Paginas/2013B214. aspx

Sayadi, J. (enero-junio, 2001). Agroturismo y desarrollo rural: situación actual, potencial y estrategias en zonas de montaña del Sureste español. Cuadernos de Turismo, 7, 131-157.

Téllez Cortés, C. (2 de octubre de 2016). Alista Peña Nieto un decreto para declarar el 16 de noviembre como Día Nacional de la Gastronomía. Crónica, 6.

Tomás, A. e Iglesias, C. (4 de octubre de 2013). "Foodies”, pasión por la comida. La Vanguardia. Recuperado de http://www.lavanguardia. com/20131004/54388403009/foodies-pasion-por-la-comida.html

Organización de las Naciones Unidas para la Educación, la Ciencia y la Cultura (s. f.). Comprender las industrias creativas. Las estadísticas como 
apoyo a las políticas públicas (pp. 1-7). Recuperado de http://portal. unesco.org/culture/es/files/30850/11467401723cultural_stat_es.pdf/ cultural_stat_es.pdf

Organización de las Naciones Unidas para la Educación, la Ciencia y la Cultura (2005). Hacia las sociedades del conocimiento. Informe mundial de la Unesco. París: Autor.

Organización de las Naciones Unidas para la Educación, la Ciencia y la Cultura (2015). La cocina tradicional mexicana, cultura comunitaria, ancestral y viva-El paradigma de Michoacán. Recuperado de http://www. unesco.org/culture/ich/index.php?lg $=$ es\&pg $=00011 \& R L=00400$

Ward, B. (18 de marzo de 2013). Millennials are becoming the foodie generation. StarTribune. Recuperado de http://www.startribune.com/lifestyle/taste/198510941.html 
